Radiologe $2021 \cdot 61: 880-887$ https://doi.org/10.1007/s00117-021-00909-0 Angenommen: 17. August 2021 Online publiziert: 20 . September 2021 (c) Springer Medizin Verlag $\mathrm{GmbH}$, ein Teil von Springer Nature 2021

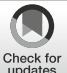

\title{
COVID-19: Epidemiologie und Mutationen
}

\section{Ein Update}

\author{
Christoph J. Hemmer · M. Löbermann · E. C. Reisinger
}

Abteilung für Tropenmedizin und Infektionskrankheiten, Universitätsmedizin Rostock, Rostock, Deutschland

\section{Zusammenfassung}

Mutationen von SARS-CoV-2 können die Ausbreitungsfähigkeit und Infektiosität erhöhen sowie die Schutzwirkung von Antikörpern vermindern, die nach einer Infektion, einer Impfung oder einer Antikörpertherapie vorhanden sind. Die AlphaVariante (B.1.1.7), die zuerst in Kent/Großbritannien beobachtet wurde, hat zu einer Erhöhung des R-Werts und damit der Infektiosität um 75\% geführt. Die Wirksamkeit der in Deutschland verfügbaren Impfungen gegen SARS-CoV-2 scheint durch diese Mutationen jedoch nur geringfügig beeinträchtigt zu sein. Bei der BetaVariante (B.1.351), die zuerst in Südafrika beschrieben wurde, ist die Fähigkeit von Antikörpern, SARS-CoV-2 zu neutralisieren, vermindert. Die therapeutisch verwendeten monoklonalen Antikörper Bamlanivimab und Etesivimab sind unwirksam. Der Impfstoff von AstraZeneca hat gegenüber leichten und mittelschweren Erkrankungen durch die Beta-Variante fast keine Schutzwirkung. Die Gamma-Variante (P.1 oder B.1.1.28.1), die zuerst in Brasilien gefunden wurde, ist wahrscheinlich um den Faktor 1,7 bis 2,6 stärker übertragbar als zuvor in Brasilien zirkulierende Virusstämme. Neben der Infektiosität scheint auch das Sterberisiko bei der Gamma-Variante um den Faktor 1,2 bis 1,9 bei Erwachsenen und bei jüngeren Patienten um den Faktor 5 bis 8 erhöht zu sein. Die Delta-Variante (B.1.617), die zuerst in Indien beschrieben wurde, ist inzwischen in den meisten Ländern dominierend. Ihre Infektiosität ist gegenüber der zuvor dominierenden Alpha-Variante um etwa $50 \%$ erhöht, die Schutzwirkung der verfügbaren Impfungen vor symptomatischen Erkrankungen kann vermindert sein (BioNTech: Delta-Variante 88\%, Alpha-Variante 93,7\%; AstraZeneca: Delta-Variante $67 \%$, Alpha-Variante 74,5\%). Ferner ist der Krankheitsverlauf bei der Delta-Variante oft schwerer als beim Wildtyp. Erkrankungen durch die Delta-Variante verlaufen bei Geimpften leichter als bei Ungeimpften, und tödliche Verläufe sind wesentlich seltener. Eine hohe Impfquote ist unerlässlich, um einer Herdenimmunität möglichst nahezukommen und die Pandemie unter Kontrolle zu bringen. Selbst bei verminderter Schutzwirkung gegenüber leichten und mittelschweren Erkrankungen bieten Impfungen in der Regel einen ausgezeichneten Schutz gegenüber lebensbedrohlichen und tödlichen Krankheitsverläufen.

\section{Schlüsselwörter}

SARS-CoV-2 - Virusvarianten · Infektiosität · Impfung $\cdot$ Krankheitsverlauf

Die COVID-19-Pandemie hat bis zum 31. Juli 2021 über 197 Mio. Erkrankungen verursacht und ca. 4,2 Mio. Menschenleben gefordert [1]. Das SARS-CoV-2 gelangt in die Wirtszellen, indem es mit der "receptorbinding domain" (rezeptorbindende Domäne, RBD) seines Spike-Proteins (S-Proteins) am ACE2-Rezeptor von Wirtszellen andockt. Dies bedeutet, dass Mutationen des viralen Spike-Proteins vor allem dann, wenn sie die RBD betreffen, Einfluss auf die Infektionstüchtigkeit des Virus haben und dass Mutationen, die das Andocken des Virus an seine Wirtszellen verbessern, die Infektiosität und den klinischen Verlauf beeinflussen. So war z.B. das Auftreten der Mutation B1.1.7 (Alpha-Variante) in der englischen Region Kent mit einer 
Hier steht eine Anzeige.

黑 Springer 
Erhöhung der Infektiosität des Virus und einem deutlichen Anstieg der Fallzahlen verbunden [2]. Inzwischen ist die AlphaVariante in Europa weitgehend durch die noch infektiösere Delta-Variante verdrängt worden.

Es gibt mittlerweile zahlreiche Hinweise darauf, dass sowohl eine vorausgegangene Infektion als auch die seit Ende 2020 verfügbaren Impfungen die weitere Ausbreitung von COVID-19 behindern und vor Infektionen, Erkrankungen, schweren Verläufen und Tod durch COVID-19 schützen können. Jedoch beeinflussen Mutationen von SARS-CoV-2 nicht nur die Infektiosität und Ausbreitungsfähigkeit dieses Virus, sondern auch die Schutzwirkung von Antikörpern, die nach einer Infektion, einer Impfung oder nach einer Antikörpertherapie vorhanden sind.

\section{Mutante „alpha“ (B.1.1.7, „Kent"- oder „Britische Mutante")}

Im Dezember 2020 fiel in der britischen Grafschaft Kent auf, dass die Zahl der Neuinfektionen mit COVID-19 nicht abnahm, obwohl dort zur Bekämpfung der Pandemie dieselben Einschränkungen eingeführt worden waren wie im übrigen England [2]. Hierzu hat wahrscheinlich die Alpha-Variante (auch als B.1.1.7 bzw. Variant of Concern 202012/01 bezeichnet) entscheidend beigetragen. Diese Variante weist gegenüber dem bis dahin vorherrschenden Stamm von SARS-CoV-2 insgesamt 23 Mutationen auf, davon mindestens 8 , die das Spike-Protein des Virus betreffen. Mindestens 2 von diesen verstärken die Fähigkeit des viralen Spike-Proteins, an seinen Rezeptor ACE2 auf der Wirtszelle zu binden. Es handelt sich um die Mutation N501Y (Austausch von Tyrosin gegen Asparagin an Position 501) sowie um die Deletion HV69-70 (Deletion von Histidin und Valin an den Positionen 69 und 70). Zusammen bewirken diese Mutationen eine Verstärkung der Übertragbarkeit um $75 \%$ [3]. Eine dritte Mutation, P681H (Ersatz von Prolin durch Histidin an Position 681), scheint ebenfalls von Bedeutung zu sein.

Die Verstärkung der Übertragbarkeit schlägt sich in einer Erhöhung der Reproduktionszahl (R) nieder. Dies bedeutet, dass Maßnahmen, die den R-Wert des ursprünglichen Virusstamms unter 1 drücken und damit dessen Ausbreitung stoppen können für die stärker übertragbare Variante oft nicht mehr ausreichen, und dass neue Varianten eine Verschärfung der Kontrollmaßnahmen erfordern.

Während erste Untersuchungen nahelegten, dass der Einfluss der Alpha-Variante auf den Krankheitsverlauf eher gering ist [3], ergaben andere Untersuchungen eine Erhöhung des Risikos für einen tödlichen Verlauf von 0,6 auf 0,9\% [4]. Hierzu könnte die Evasion der innaten Immunabwehr durch diese Variante beitragen [5].

Die Alpha-Variante (B.1.1.7) hat sich seit dem Jahreswechsel 2020/2021 in ganz Europa stark ausgebreitet. In Deutschland machte sie laut Robert-Koch Institut (RKI) in der 16. Kalenderwoche (19.-25.04.2021) bereits $90 \%$ der nachgewiesenen SARSCoV-2-Infektionen aus [6].

Ein Einfluss der Alpha-Variante auf die Wahrscheinlichkeit, sich ein zweites Mal mit SARS-CoV-2 zu infizieren, konnte nicht gezeigt werden [7]. Auch die Fähigkeit von Rekonvaleszentenplasma zur Virusneutralisation war gegenüber der Variante B.1.1.7 nicht nennenswert vermindert, obwohl diese Variante gegenüber bestimmten monoklonalen Antikörpern vermindert empfindlich war [8].

Die Schutzwirkung der verfügbaren Impfungen gegen COVID-19 scheint für die Alpha-Variante nicht vermindert zu sein. Zumindest ist die neutralisierende Wirkung des Plasmas von Personen, die mit den Impfstoffen von BioNTech/Pfizer, Moderna oder Novavax geimpft worden sind, im Pseudovirus-Neutralisationstest gegenüber der Alpha-Variante allenfalls um den Faktor 2 geringer als gegenüber dem bis dahin gängigen Wildvirusstamm $[8,9]$. Dieser Unterschied ist höchstwahrscheinlich zu gering, um die praktische Schutzwirkung der Impfungen zu beeinflussen. Die Plasmen von mit dem Impfstoff von AstraZeneca Geimpften haben gegenüber der Alpha-Variante eine um den Faktor 9-10 geringere Neutralisationswirkung, als gegenüber einem Virusstamm ohne die für die Alpha-Variante typischen Mutationen. Dieser Unterschied bleibt jedoch wahrscheinlich ohne Bedeutung, da die Antikörpertiter in ihrer absoluten Höhe trotzdem noch für eine Schutzwirkung ausreichen.
Die hohe Schutzwirkung der Impfung gegenüber der Alpha-Variante zeigt sich im Übrigen an der zunächst höchst erfolgreichen Bekämpfung der Pandemie mit dem Impfstoff von BioNTech-Pfizer in Israel, wo zwischen dem 24.01. und dem 03.04.2021 der Anteil der Alpha-Variante bei $94,5 \%$ gelegen hat [10]. Dabei hatte die Impfung eine Schutzwirkung von $97 \%$ gegen Erkrankung und Tod und von $94 \%$ gegen die Infektion.

Ähnliches gilt für Großbritannien, wo per 19.05.2021 etwa 94\% aller COVID-19Fälle auf der Alpha-Variante beruhten [11]. Dabei hatte der Impfstoff von AstraZeneca, derin Großbritannien neben dem Impfstoff von BioNTech-Pfizer in großem Umfang verwendet wird, eine Schutzwirkung von $81,5 \%$ gegen Nicht-B.1.1.7-Stämme und 70,4\% gegen die Alpha-Variante (B.1.1.7; [12]).

Spätestens seit Juni 2021 hat in Großbritannien die zuerst nur in Indien beobachtete Delta-Variante von SARS-CoV-2 die Alpha-Variante weitestgehend verdrängt (s. unten).

\section{Mutante "beta" (B.1.351 oder "Südafrikanische Mutante")}

Die Beta-Variante von SARS-CoV-2 (B.1.351, auch als 501.V2 bezeichnet) wurde zum ersten Mal im Oktober 2020 im östlichen Südafrika identifiziert. Bis zum 27. Juni 2021 wurden weltweit 22.332 Infektionen mit dieser Variante gemeldet, davon 4105 in Südafrika (von insgesamt 1,8 Mio. Fällen; [13]). Weitere Länder mit Fallzahlen über 1000 sind Schweden (2205), Deutschland (2147), die USA (1968), Frankreich (1815) und Finnland (1092). In Südafrika gingen bereits im Dezember 2020 insgesamt $87 \%$ der typisierten SARS-CoV2-Infektionen auf das Konto der BetaVariante [14]. Diese Variante hat sicherlich wesentlich zu der zweiten COVID19-Welle ab November 2020 in Südafrika beigetragen. Sie ist wahrscheinlich um den Faktor 1,5 kontagiöser als vorher in Südafrika zirkulierende Virusstämme [15].

Ferner ist die Beta-Variante in weiteren Ländern für die Mehrzahl der COVID-19Fälle verantwortlich. Dies betrifft insbesondere Sambia, wo ab Dezember 2020 ein massiver Anstieg der Fallzahlen zu beobachten ist, aber auch weitere afrikanische 
Länder einschließlich Botswana, Lesotho, Mosambik und Simbabwe [16]. Außerhalb Afrikas ist Bangladesch zu nennen, wo diese Variante zu einer Verdopplung der effektiven Reproduktionsrate von etwa 1,0 auf 2,0 und ab März 2021 zu einer exponentiellen Ausbreitung von COVID-19 geführt hat [17].

Ferner dürften die Fallzahlen in Tansania, welches seit April 2020 keine COVIDFallzahlen mehr veröffentlicht, sehr hoch sein, zumal der am 17.03.2021 an COVID-19 verstorbene Präsident Magafuli am Sonntag, dem 07. Juni 2020, unzutreffenderweise verkündet hatte, in Tansania sei die Epidemie überwunden [18]. Die Beta-Variante ist bereits mehrfach bei aus Tansania kommenden Reisenden identifiziert worden $[19,20]$.

Acht Mutationen der Beta-Variante betreffen die Spike-Region. Drei dieser Mutationen betreffen die rezeptorbindende Domäne (RBD) des Spike-Proteins, daher wird angenommen, dass sie die Ausbreitung und Pathogenität des Virus beeinflussen. Dabei handelt es sich um die Mutationen N501Y (Austausch von Asparagin gegen Tyrosin an Position 501), E484K (Austausch von Glutaminsäure gegen Lysin) und K417N/T (Austausch von Lysin gegen Asparagin oder Threonin; [21]). Ferner könnte die Verkürzung des Spike-Proteins durch Deletion von 242 Basenpaaren, die das N-terminale Ende des Spike-Proteins codieren, die vermutete Immunität nach Infektionen beeinträchtigen, da auch eine Antikörperantwort gegen das N-terminale Ende (N-terminal Domain oder NTD) des Spike-Proteins die Virusvermehrung hemmen kann [22]. Die genauen Mechanismen hierfür sind allerdings noch unklar. Die Beta-Variante hat die Mutation N501Y mit der Alpha-Variante gemeinsam. Diese Mutation verbessert die Fähigkeit des Virus, an den Rezeptor ACE2 der Wirtszelle zu binden. Die beiden anderen Mutationen beeinträchtigen die Virusneutralisation durch Sera von Rekonvaleszenten und Geimpften, was dem Virus die Verbreitung erleichtern dürfte [23].

Bisher liegen nur wenige Informationen zur Virulenz der Beta-Variante vor. In Südafrika lag die Krankenhausletalität während der zweiten Welle (Höhepunkt Januar 2021), die - im Gegensatz zur ersten Welle (Höhepunkt Juli 2020) - fast vollständig auf der Beta-Variante beruhte, um 20\% höher als während der ersten Welle. Die höhere Letalität war dabei nicht durch die höhere Zahl der Krankenhauseinweisungen erklärt [24].

Auch wenn der Einfluss der Beta-Variante auf den klinischen Schweregrad derzeit gering zu sein scheint, so ist eine Immunevasion zu beachten. Es wurden Patienten beschrieben, die eine Reinfektion mit der Beta-Variante erlitten hatten, nachdem sie zuvor eine Erkrankung mit einem vor der Ausbreitung dieser Mutante gängigen Stamm durchgemacht hatten [25]. Eine andere Studie hat gezeigt, dass 21 von 44 Plasmen (48\%) von Patienten, die eine Erkrankung mit dem Virusstamm Wuhan-1 D614G durchgemacht hatten, in vitro keine neutralisierende Wirkung gegenüber der Beta-Variante hatten [26].

Auch die Schutzwirkung der verfügbaren COVID-19-Impfstoffe gegenüber der Beta-Variante kann vermindert sein. Insbesondere die Impfung von AstraZeneca hatte in einer südafrikanischen Studie gegenüber leichten und mittelschweren Erkrankungen durch die Beta-Variante eine Schutzwirkung von nur 10,4\%, während die Schutzwirkung bei allen untersuchten Geimpften, 21,9\% betrug [27]. Bei 11 von 13 geimpften Studienteilnehmern, bei denen Serum-Antikörper mittels Neutralisationstest bestimmt wurden, waren keine schützenden Antikörper gegen die BetaVariante nachweisbar. Dies führte dazu, dass Südafrika den Impfstoff von AstraZeneca von seinem Impfprogramm ausschloss.

In einer weiteren Studie rief dagegen die Impfung mit dem BioNTech-Impfstoff bei allen Teilnehmern ausreichend neutralisierende Antikörpertiter gegen mehrere Virusvarianten hervor [28]. Die neutralisierende Aktivität gegen die Beta-Variante war zwar - verglichen mit der Aktivität gegen das vorher zirkulierende Standardvirus - vermindert, aber immer noch deutlich nachweisbar. Daten aus Qatar, wo sowohl die Alpha- als auch die Beta-Variante verbreitet ist, haben eine Schutzwirkung der BioNTech-Impfung gegenüber der Alpha-Variante von $89,5 \%$ und gegenüber der Beta-Variante von $75 \%$ in Bezug auf die Infektion gezeigt [29]. Der Schutz vor schweren und tödlichen Verläufen betrug $97,4 \%$.
Eine andere Studie ergab 6 bis 8 Monate nach der Grundimmunisierung mit 2 Moderna-Impfungen das Fehlen protektiver Titer gegen die Beta-Variante und die Gamma-Variante (P.1, Brasilien), allerdings wurden nach einer Booster-Impfung mit dem originalen Moderna-Impfstoff protektive Titer gegen diese beiden Varianten erreicht [30]. Ein Booster mit einem an die Varianten angepassten Impfstoffergab höhere Titer als mit dem Original-Impfstoff.

Der Impfstoff von Johnson \& Johnson schützt laut einer Zulassungsstudie zu $66 \%$ gegen mittelschwere bis schwere Verläufe von COVID-19. Gegen lebensbedrohliche Verläufe beträgt der Schutz $76,7 \%$ zwei Wochen nach der Impfung und 85,4\% vier Wochen nach der Impfung [31]. In Südafrika, wo zum Zeitpunkt der Studie die BetaVariante vorherrschte, betrug laut dieser Studie die Schutzwirkung gegenüber mittelschweren bis schweren Verläufen $52 \%$ nach 2 Wochen und $64 \%$ nach 4 Wochen.

\section{Mutante "gamma" (P.1 oder "Brasilianische Mutante", B.1.1.28.1)}

Ab Mai 2020 war die Amazonas-Region in Brasilien von einer massiven Welle von COVID-19 betroffen; dies hatte bis Oktober 2020 zu einer "attack rate" von $75 \%$ der Bevölkerung geführt [32]. Am 06. Januar 2021 wurden im Nationalen Institut für Infektionskrankheiten Japans bei 4 Reisenden, die 4 Tage zuvor Manaus besucht hatten, eine neue Variante von SARS-CoV-2 mit 17 Mutationen auf Aminosäurenebene identifiziert, von denen 3 die RBD des Virus betreffen und das Anbinden des Virus an den ACE2-Rezeptor der Wirtszelle begünstigen [33]. Dies sind E484K (Austausch von Glutaminsäure gegen Lysin), K417T (Austausch von Lysin gegen Threonin) und N501Y (Austausch von Asparagin gegen Tyrosin). Diese Mutationen sind auch bei der Beta-Variante vorhanden, und die Mutation N501Y findet sich auch bei der Alpha-Variante.

Ferner ist in Brasilien eine als P.2 bezeichnete Mutante beobachtet worden, die - anders als P. 1 - im Bereich der RBD nur die Mutation E484K, nicht jedoch die beiden anderen Mutationen trägt [33].

Die Gamma-Variante (Variante P.1) wurde in Brasilien in keiner Probe aus dem Zeit- 
raum zwischen März und November 2020, jedoch in 35 von 67 Proben von Dezember 2020 (52,2\%) und in 41 von 48 Proben $(85,4 \%)$ von Januar 2021 gefunden [33]. Gleichzeitig stieg die Zahl der täglichen Neuerkrankungen deutlich an [34]. Während sie auf dem Höhepunkt der ersten Erkrankungswelle Ende Juli und Anfang August 2020 zwischen 23.000 und 71.000 lag und bis November 2020 auf 10.000 bis 23.000 abgenommen hatte, wurden Ende Dezember 2020 zwischen 21.000 und 70.000 Neuerkrankungen und Ende März 2021 zwischen 53.000 und 98.000 Neuerkrankungen gemeldet. Modellrechnungen ergaben, dass die Gamma-Variante um den Faktor 2,6 stärker übertragbar ist, als die vorher in Brasilien zirkulierenden Virusstämme [35]. Diese Modellrechnungen ergaben auch, dass zwischen November 2020 und Januar $202128 \%$ der Fälle in Manaus auf Reinfektionen mit der GammaVariante beruhten. Eine andere Schätzung ergab, dass die Gamma-Variante zwischen 1,7- und 2,4-fach besser übertragbar ist, als die vorher zirkulierenden SARS-CoV-2Stämme. Dabei hat eine vorherige SARSCoV-2-Infektion mit einem anderen Virusstamm gegenüber einer Reinfektion mit der Gamma-Variante nur zwischen 54 und $79 \%$ der Schutzwirkung, verglichen mit der Schutzwirkung gegen Reinfektion mit vorher dominierenden Virusstämmen [36].

Außerdem ist die Gefahr, an COVID19 zu versterben, bei der Gamma-Variante wahrscheinlich um den Faktor 1,2 bis 1,9 erhöht [36]. Bei jüngeren Patienten ohne Vorerkrankungen kann das Sterberisiko gegenüber den bisher dominierenden Virusstämmen um den Faktor 5 bis 8 erhöht sein [37].

Laboruntersuchungen zeigen, dass die Hemmwirkung von Rekonvaleszentenserum sowie der monoklonalen Antikörperpräparate Bamlanivimab und Etesevimab im Neutralisationstest gegenüber der Beta-Variante und der Gamma-Variante um den Faktor 3,8 bis 4,8 vermindert ist [38, 39]. Dies gilt auch für Sera von mit BioNTtech- oder Moderna-Geimpften [38]. Eine andere Studie berichtet eine Reduktion der Neutralisationswirkung von Impfseren gegenüber der Gamma-Variante um den Faktor 2,6 für BioNTech und 2,9 für AstraZeneca [40].
Klinische Felddaten über die Präventionswirkung der verfügbaren Impfungen gegenüber der Gamma-Variante sind derzeit noch nicht verfügbar. Trotzdem erscheint die Annahme gerechtfertigt, dass diese Impfstoffe auch unter realen Bedingungen noch eine gute Schutzwirkung auch gegenüber der Gamma-Variante entfalten, da selbst die gegenüber der Gamma-Variante verminderte Antikörper-Antwort wahrscheinlich immer noch für eine Schutzwirkung ausreichen [28]. Außerdem trägt die schwierig zu messende zellvermittelte Immunantwort zur Wirkung von Impfstoffen bei.

\section{Mutante "delta" (B.1.617.2, „Indische Mutante")}

Anfang Oktober 2020 wurde aus Maharashtra/Indien eine neue SARS-CoV-2-Variante an die "Global Initiative on Sharing All Influenza Data“ (GISAID) gemeldet [41]. Diese Variante, inzwischen unter dem Namen „Delta-Variante“ bekannt, erhielt gemäß dem taxonomischen Ordnungssystem „Phylogenetic Assignment of Named Global Outbreak Lineages" (PANGOLIN) zunächst die Bezeichnung B.1.617. Sie zeichnet sich u.a. durch die Mutationen E484Q (Glutaminsäure zu Glutamin) und L452R (Leucin zu Arginin) im Bereich des viralen Spike-Proteins aus [42]. Beide Mutationen bewirken eine stärkere Bindung des Virus an den ACE2-Rezeptor an die Wirtszellen. Weiterhin werden die Mutationen D614G (Asparaginsäure zu Glycin) und P681R (Prolin zu Arginin) beobachtet, die ebenfalls wahrscheinlich die Infektiosität erhöhen. Dabei wird die Variante B.1.617 in 3 Untervarianten aufgeteilt, B.1.617.1, B.1.617.2 und B.1.617.3. Die größte Rolle spielt in Indien derzeit die Untervariante B.1.617.2, die inzwischen als die eigentliche DeltaVariante angesehen wird. Diese wurde am 03.05.2021 bei 24 von 28 sequenzierten Proben gefunden [43]. Am 26.02.2021 war diese Variante dagegen noch bei keiner von 101 sequenzierten Proben nachgewiesen worden. Parallel zu dieser Ausbreitung der Delta-Variante wurde in Indien eine zweite COVID-19-Welle mit über 355.000 gemeldeten neuen Fällen täglich beobachtet [44]. Dagegen lag auf dem Höhepunkt der ersten Krankheitswelle in Indien im September 2020 die Zahl der täglich ge- meldeten neuen Fälle an keinem Tag über 100.000. Ein Teil des Anstieges der Fallzahlen dürfte auch mit religiösen und spirituellen Feierlichkeiten zusammenhängen. So wurde im April 2021 im zeitlichen Zusammenhang mit dem Kumbh-Mela-Fest, das alle 12 Jahre stattfindet, im indischen Bundesstaat Uttarakhand ein Anstieg der Erkrankungszahlen um den Faktor 18 beobachtet [45]. Allerdings ist die Zahl der aus Indien gemeldeten Neuerkrankungen nach dem 09. Mai 2021 wieder gesunken, ohne dass der Grund hierfür erkennbar ist.

In England lag zwischen dem 07. und dem 21. Juni 2021 der Anteil der DeltaVariante an den sequenzierten und genotypisierten SARS-CoV-2-Proben zwischen 92 und $95 \%$. Nach Schätzungen der britischen Regierung ist die Delta-Variante etwa $50 \%$ stärker übertragbar und verbreitet sich etwa um $13 \%$ schneller als die vorher in Großbritannien vorherrschende Alpha-Variante [46]. In England war bis zum 07. Mai 2021 von 509 Patienten mit gesicherter Infektion durch die Delta-Variante kein Patient verstorben [47]. Insgesamt verstarben zwischen dem 01. Februar und dem 21. Juni 2021 in England 4262 von 219.570 Patienten (1,9\%) mit der AlphaVariante und 117 von 50.283 Patienten $(0,1 \%)$ mit der Delta-Variante [48].

Eine neuere retrospektive Auswertung von 211.197 COVID-19-Fällen aus Kanada ergab, dass bei Varianten mit der Mutation N501Y, also Alpha, Beta und Gamma, das Risiko für eine Krankenhausaufnahme um $59 \%$, für eine Intensivtherapie um $105 \%$, und für das Versterben um $61 \%$ erhöht ist. Für die Delta-Variante ergibt sich dagegen eine Erhöhung des Risikos für eine Krankenhausaufnahme um $120 \%$, für eine Intensivtherapie um $287 \%$ und für das Versterben um 137\% [49]. Eine Studie aus Singapur ergab für die Delta-Variante eine Erhöhung des Risikos für Sauerstoffbedarf, Intensivtherapie oder Tod um den Faktor 4,90 („adjusted odds ratio“; 95\% Konfidenzintervall [KI] 1,43-30,78), gegenüber dem Wildtyp [50]. Ferner war die DeltaVariante mit höheren Viruslasten und einer langsameren Abnahme der Viruslast im Verlauf assoziiert als der Wildtyp. Eine schottische Studie ergibt eine um den Faktor 1,85 erhöhte Wahrscheinlichkeit einer Krankenhausaufnahme bei der Delta- 
Variante, verglichen mit der zuvor dominierenden Alpha-Variante [51].

Rekonvaleszenten-Plasma von Wildtyp-Patienten zeigte im Neutralisationstest gegen die Delta-Variante eine um den Faktor 2, und gegen die Beta-Variante eine um den Faktor 6 schwächere Neutralisationswirkung als gegen den Wildtyp [52]. Es ist bisher nicht bekannt, ob eine der 3 Typen der Delta-Variante (B.1.617.1 oder .2, oder .3) auch vermehrt Reinfektionen verursacht. Auffällig ist jedoch, dass die DeltaVariante - wie auch die Beta-Variante gegenüber dem therapeutisch verwendeten Antikörper Bamlanivimab resistent ist, während der meist in Kombination mit Bamlanivimab verwendete Antikörper Etesivimab in vitro gegen die BetaVariante unwirksam, jedoch gegen die Delta-Variante wirksam ist [52]. Plasmen von mit dem BioNTech-Impfstoff Geimpften hatten, verglichen mit dem SARSCoV-2 Wildtyp, eine um den Faktor 11 verminderte Schutzwirkung gegenüber der Beta-Variante und um den Faktor 3 verminderte Schutzwirkung gegenüber der Delta-Variante. Diese Reduktion ist jedoch in vivo mit einem immer noch ausreichenden Impfschutz gegenüber der Delta-Variante und, in geringerem Maße, gegenüber der Beta-Variante vereinbar.

Impfdaten aus Großbritannien, wo nach Indien die höchste Zahl von Infektionen mit der Delta-Variante beobachtet werden, zeigen für die BioNTech-Impfung einen Schutz vor symptomatischer Erkrankung von $93,7 \%$ gegenüber der Alpha-Variante und von $88,0 \%$ gegenüber der DeltaVariante [53]. Für die Impfung von AstraZeneca ergibt sich ein Schutz von $74,5 \%$ gegenüber der Alpha-Variante und von $67,0 \%$ gegenüber der Delta-Variante [53]. Eine Virusneutralisationsstudie mit Seren von Rekonvaleszenten und von mit den Impfstoffen von BioNTech oder Moderna Geimpften ergab gegenüber dem WildtypStamm eine um den Faktor 7 verminderte Neutralisationswirkung für die mit der Delta-Variante B.1.617.2 eng verwandten Variante B.1.617.1. Allerdings waren alle Seren von geimpften Personen trotzdem in der Lage, das Virus zu neutralisieren [54].

Eine Studie aus Singapur ergab, dass bei Erkrankungen durch die Delta-Variante nach zuvor erfolgter Impfung mit einem mRNA-Impfstoff deutlich seltener Sauer- stoff benötigt wurde als bei Ungeimpften (,adjusted odds ratio“: 0,07; $95 \% \mathrm{KI}$ $0,015-0,335 ; p=0.001)$. Während die initialen $C(t)$-Werte bei geimpften und ungeimpften Delta-Variante-Patienten ähnlich lagen, nahm die Viruslast bei Geimpften schneller ab als bei Ungeimpften [55].

\section{Ausblick}

Die in Deutschland verfügbaren Impfstoffe bieten auch gegen die neuen Virusvarianten Schutz. Das Auftreten von weiteren Problemvarianten kann vor allem dadurch verhindert werden, dass man die Infektionszahlen niedrig hält. Dazu ist die möglichst vollständige Durchimpfung der Weltbevölkerung geeignet. Dies gilt insbesondere dann, wenn neue Varianten auftreten werden. Es liegt daher im eigenen Interesse der reichen Länder, den ärmeren Ländern ausreichend Impfstoffe zur Verfügung zu stellen, da dies nicht nur in den armen Ländern Leben rettet, sondern auch dem Entstehen und Übergreifen neuer Varianten entgegenwirkt.

\section{Fazit für die Praxis}

- Die Delta-Variante führt per se vermehrt zu Krankenhausaufnahmen, zu Intensivtherapie und zu Todesfällen.

- Die verfügbaren Impfungen haben auch gegenüber den jetzt aufgetretenen Virusvarianten eine gute Schutzwirkung.

- Durch die konsequente Impfung von älteren Menschen und Risikopatienten können Intensivstationsaufenthalte und Todesfälle deutlich reduziert werden, wie dies derzeit am Beispiel Großbritanniens und Israels eindrucksvoll gezeigt wird.

- Eine hohe Impfquote trägt auch entscheidend dazu bei, langfristige Gesundheitsschäden zu verhindern.

- Daher sollte unbedingt eine möglichst hohe Durchimpfung der Bevölkerung angestrebt werden.

\section{Korrespondenzadresse}

PD Dr. med. Christoph J. Hemmer Abteilung für Tropenmedizin und Infektionskrankheiten, Universitätsmedizin Rostock

Ernst-Heydemann-Straße 5, 18057 Rostock, Deutschland

Christoph.Hemmer@uni-rostock.de

\section{Einhaltung ethischer Richtlinien}

Interessenkonflikt. C.J. Hemmer, M. Löbermann und E.C. Reisinger geben an, dass kein Interessenkonflikt besteht.

Für diesen Beitrag wurden von den Autoren keine Studien an Menschen oder Tieren durchgeführt. Für die aufgeführten Studien gelten die jeweils dort angegebenen ethischen Richtlinien.

\section{Literatur}

1. https://www.worldometers.info/coronavirus/. Zugegriffen: 2. Aug. 2021

2. Kirby $T$ (2021) New variant of SARS-CoV-2 in UK causes surge of COVID-19. Lancet Respir Dis. https://doi.org/10.1016/S2213-2600(21)00005-9

3. Leung K, Shum MHH, Leung GM, Lam TTY, Wu JT (2021) Early transmissibility assessment of the N501Y mutant strains of SARS-CoV-2 in the United Kingdom, October to November 2020. Euro Surveill. https://doi.org/10.2807/1560-917. ES.2020.26.1.2002106

4. Davies NG, Jarvis CI, CMMID COVID-19 Working Group et al (2021) Increased mortality in community-tested cases of SARS-CoV-2 lineage B.1.1.7. Nature 593:270-274

5. Thorne LG, Bouhaddou M, Reuschl AK et al (2021) Evolution of enhanced innate immune evasion by the SARS-CoV-2 B.1.1.7 U.K. variant. BioRxiv. https://doi.org/10.1101/2021.06.06.446826 (Preprint)

6. RKI Bericht zu Virusvarianten von SARS-CoV-2 in Deutschland. https://www.rki.de/DE/Content/ InfAZ/N/Neuartiges_Coronavirus/DESH/Bericht_ VOC_2021-04-28.pdf?_blob=publicationFile (Erstellt:28.Apr. 2021).Zugegriffen: 30. Mai 2021

7. Graham MS, SudreCH,MayAetal (2021)Changes in symptomatology, reinfection, and transmissibility associated with the SARS-CoV-2 variant B.1.1.7: an ecological study. Lancet 6:E335-E345. https://doi. org/10.1016/S2468-2667(21)00055-4

8. Wang P, Nair MS, Liu L et al (2021) Antibody resistance of SARS-CoV-2 variants B.1.351 and B.1.1.7. Nature 593:130-135. https://doi.org/10. 1038/s41586-021-03398-2

9. Shen X, Tang H, McDanal C et al (2021) SARSCoV-2 variant $B \cdot 1.1 .7$ is susceptible to neutralizing antibodies elicited by ancestral spike vaccines. Cell Host Microbe 29:529-539.e3. https://doi.org/10. 1016/j.chom.2021.03.002

10. Haas EJ, Angulo FJ, McLaughlin JM et al (2021) Impact and effectiveness of mRNA BNT162b2 vaccine against SARS-CoV-2 infections and COVID19 cases, hospitalisations, and deaths following a nationwide vaccination campaign in Israel: an observational study using national surveillance data. Lancet 397:P1819-P1829. https://doi.org/ 10.1016/S0140-6736(21)00947-8

11. Public Health England (2021) Research and analysis. Variants: distribution of cases data. https://www.gov.uk/government/publications/ covid-19-variants-genomically-confirmed-casenumbers/variants-distribution-of-cases-data (Erstellt:27. Mai 2021).Zugegriffen:30. Mai 2021

12. Emary et al (2021) Efficacy of ChAdOx1 nCoV-19 (AZD1222) vaccine against SARS-CoV-2 variant of concern 202012/01 (B.1.1.7): an exploratory analysis of a randomised controlled trial. Lancet 397(10282):P1351-P1362. https://doi.org/10. 1016/S0140-6736(21)00628-0 
13. https://cov-lineages.org/global_report_B.1.351. html.Zugegriffen:30. Mai 2021

14. Karim SSA, de Oliveira T (2021) New SARS-CoV-2 Variants-Clinical, Public Health, and Vaccine Implications. N EnglJ Med 384:1866-1868. https:// doi.org/10.1056/NEJMc2100362

15. Pearson CAB, Russell TW, Davies N et al (2021) Estimates of severity and transmissibility of novel SARS-CoV-2 variant 501Y.V2 in South Africa. CMMID Repository. https://cmmid.github. io/topics/covid19/sa-novel-variant.html (under review).Zugegriffen:30. Mai 2021

16. Dolgin E Coronavirus Variant threatens global recovery. Nature. News Feature. https://www.nature. com/articles/d44148-021-00021-4.Zugegriffen: 14. Apr. 2021

17. Brum E, Saha S, Sania A et al (2021) Surging COVID-19 in Bangladesh driven by B.1.351 variant. medRxiv. https://doi.org/10.1101/2021.04.27. 21255706

18. CNN https://edition.cnn.com/2020/06/09/africa/ tanzania-president-covid-claims/index.html (Erstellt:9. Juni 2020).Zugegriffen:30. Mai 2021

19. https://edition.cnn.com/2021/02/11/africa/ tanzania-covid-cases-surge-int//index.html. Zugegriffen: 30. Mai 2021

20. Yadav PD (2021) Imported SARS-CoV-2 V501Y.V2 variant (B.1.351) detected in travelers from South Africa and Tanzania to India. Travel Med Infect Dis. https://doi.org/10.1016/j.tmaid.2021.102023

21. Gupta RK (2021) Will SARS-CoV-2 variants of concern affect the promise of vaccines? Nat Rev Immunol 21:340-341

22. Chi X, Yan R, Zhang J et al (2020) A neutralizing human antibody binds to the $\mathrm{N}$-terminal domain of the Spike protein of SARS-CoV-2. Science 369:650-655. https://doi.org/10.1126/science. abc6952

23. Planas D et al (2021) Sensitivity of infectious SARSCoV-2 B.1.1.7 and B.1.351 variants to neutralizing antibodies. Nat Med 27:917-924

24. Jassat W, Mudara C, Ozougwu L et al (2021) Difference in mortality among individuals admitted to hospital with COVID-19 during the first and second waves in South Africa: a cohort study. Lancet. https://doi.org/10.1016/S2214-109X(21)00289-8

25. Staub T, Arend V, de la Vega ECL et al (2021) Case series of four re-infections with a SARS-CoV-2 B.1.351 variant, Luxembourg, February 2021. Euro Surveill. https://doi.org/10.2807/1560-7917.ES. 2021.26.18.2100423

26. Wibmer CK, Ayres F, Hermanus $T$ et al (2021) SARS-CoV-2 501Y.V2 escapes neutralization by South African COVID-19 donor plasma. Nat Med 27:622-625

27. Mahdi SA, Baillie V, Cutland CL et al (2021) Efficacy of theChAdOx $1 \mathrm{nCoV}$-19COVID-19Vaccineagainst the B.1.351 Variant. N Eng J Med 384:1885-1898. https://doi.org/10.1056/NEJMoa2102214

28. Liu Y, Liu J, Xia H et al (2021) Neutralizing activity of BNT162b2-elicited serum. N Eng J Med 384:1466-1468. https://doi.org/10.1056/ NEJMc2102017

29. Abu-Raddad LJ, Butt AA (2021) Effectiveness of the BNT162B2 COVID-19 vaccine against the B.1.1.7 and B.1.351 variants. N Eng J Med 385:187-189. https://doi.org/10.1056/NEJMc2104974

30. Wu K, Choi A, Koch M et al (2021) Preliminary analysis of safety and Immunogenicity of a SARScoV-2 variant vaccine booster. MedRxiv preprint. https://doi.org/10.1101/2021.05.05.21256716

31. Sadoff J, Gray G, Vandebosch A et al (2021) Safety and efficacy of a single dose Ad26.COV2.S vaccine

\section{COVID-19: epidemiology and mutations. An update}

Mutations of severe acute respiratory syndrome coronavirus 2 (SARS-CoV-2) can enhance the spread and the infectiousness and decrease the protective effect of antibodies present after infection, vaccination or antibody treatment. The alpha variant (B.1.1.7), first seen in Kent/United Kingdom, has increased the R-value and therefore the infectiousness by $75 \%$; however, the effectiveness of the vaccines against SARSCoV-2 available in Germany seems to be only slightly impaired by these mutations. In the case of the beta variant (B.1.351), first described in South Africa, the neutralization ability of antibodies towards SARS-CoV-2 is decreased. The monoclonal antibodies bamlanivimab and etesivimab, which are used therapeutically, are ineffective. The AstraZeneca vaccine offers almost no protection against mild or moderate disease caused by the beta variant. The gamma variant (P.1 or B.1.1.28.1), which was first found in Brazil, is probably 1.7-2.6 times more transmissible than previous virus strains circulating in Brazil. In addition to the infectiousness, the mortality risk of the gamma variant also seems to be increased between 1.2 and 1.9-fold in adults and between 5 and 8-fold in young persons. The delta variant (B.1.617), first described in India, is now dominant in most countries. It is $50 \%$ more infectious than the alpha variant, and the protective effect of vaccinations against symptomatic disease can be decreased (Biontech: delta variant 88\%, alpha variant 93.7\%; AstraZeneca: delta variant 67\%, alpha variant $74.5 \%$ ). Furthermore, the course of the disease with the delta variant is often more severe than with the wild type. Disease courses with the delta variant are less severe in vaccinated than in nonvaccinated persons, and fatal outcomes are substantially rarer. A high vaccination rate is essential in order to approach herd immunity and to bring the pandemic under control. Even where the protective effect towards mild or moderate disease is decreased, as a rule, vaccination still offers excellent protection against life-threatening and fatal disease courses.

\section{Keywords}

SARS-CoV-2 - Virus variants · Infectiousness - Vaccination · Disease severity

against COVID-19. N Eng J Med 384:2187-2201. https://doi.org/10.1056/NEJMoa2101544

32. Buss LF, Prete CA Jr, Abrahim CMM et al (2021) Three quarters attack rate of SARS-CoV-2 in the Brazilian Amazon during a largely unmitigated epidemic. Science 371:288-292. https://doi.org/ 10.1126/science.abe9728

33. Faria NR, Claro IM, Candido D et al Genomic characterisation of an emergent SARSCoV-2 lineage in manaus. Preliminary findings. Virological.org. https://virological.org/t/ genomic-characterisation-of-an-emergent-sarscov2-lineage-in-manaus-preliminary-findings/ 586 (Erstellt: 20. Jan. 2021). Zugegriffen: 1. Juni 2021

34. https://worldometers.info/coronavirus/country/ brazil.Zugegriffen:30. Mai 2021

35. Coutinho RM, Marquitti FMD, Ferreira LS et al Model-based estimation of transmissibility and reinfection of SARS-CoV-2 P.1 variant. MedRxiv 23.03.2021. https://medrxiv.org/content/ 10.1101/2021.03.03.21252706v3. Zugegriffen: 30. Mai 2021

36. Faria NR, Mellan TA, Whittaker $C$ et al (2021) Genomics and epidemiology of the P.1 SARS-CoV-2 lineage in Manaus, Brazil. Science 372:815-821. https://doi.org/10.1126/science.ahb2644

37. Freitas ARR, Lemos DRQ, Beckedorff OA, De Goes Cavalcanti LP, Siqueria AM, de Mello SRC, Barros ENC The increase in the risk of severity and fatality rate of Covid-19 in southern Brazil after the emergence of the Variant of Concern (VOC) SARSCoV-2 P.1 was greater among young adults without preexisting risk conditions. MedRxiv. https://doi. org/10.1101/2021.04.13.21255281.Zugegriffen: 30. Mai 2021

38. Wang $P$, Wang $M$, Yu J et al (2021) Increased resistance of SARS_coV-2 variant P.1 to antibody neutralization. BioRxiv. https://doi.org/10.1101/ 2021.03.01.433466

39. Emergency Use Authorization USA https://fda. gov/media/145802/download (Erstellt: 14. Mai 2021).Zugegriffen:30. Mai 2021

40. Dejnirattisai W, Zhou D, Supasa $P$ et al (2021) Antibody evasion by the P.1 strain of SARS-coV-2. Cell 184(11):2939-2954.e9. https://doi.org/10. 1016/j.cell.2021.03.055

41. https://gisaid.org/hcov19-variants/. Zugegriffen: 1.Juni 2021

42. Greany AJ, Loes AN, Crawford KHD et al (2021) Comprehensive mapping of mutations in the SARS-CoV-2 receptor-binding domain that affect recognition by polyclonal human plasma antibodies. Cell Host Microbe 29:P463-P476.e6. https:// doi.org/10.1016/j.chom.2021.02.003

43. https://outbreak.info/situation-reports? pango $=B \cdot 1.617 .2 \&$ selected $=$ IND\&loc $=$ IND . Zugegriffen: 1. Juni 2021

44. https://worldometers.info/coronavirus/country/ india.Zugegriffen: 1. Juni 2021

45. https://www.newindianexpress.com/nation/ 2021/apr/27/mahakumbh-impact-uttarakhandactive-covid-caseload-jumped-1800-in-25days-2295337.html.Zugegriffen: 28. Juni 2021

46. https://www.gov.uk/government/publications/ sage-89-minutes-coronavirus-covid-19- 
response-13-may-2021/sage-89-minutescoronavirus-covid-19-response-13-may-2021. Zugegriffen: 1. Juni 2021

47. SARS-CoV-2 variants of concern and variants under investigation in England. Technical Briefing 10. https://assets.publishing.service. gov.uk/government/uploads/system/uploads/ attachment_data/file/984274/Variants_of_ Concern VOC Technical Briefing 10 England. pdf(Erstellt: 7. Mai 2021).Zugegriffen: 1. Juni 2021

48. SARS-CoV-2 variants of concern and variants under investigation in England. Technical Briefing 17. https://assets.publishing.service. gov.uk/government/uploads/system/uploads/ attachment_data/file/984274/Variants_of Concern_VOC_Technical_Briefing_17_England. pdf (Erstellt: 25 . Juni 2021). Zugegriffen: 28. Juni 2021

49. Fisman DN, Tuite AR (2021) Progressive Increase in Virulence of Novel SARS-CoV-2 Variants in Ontario, Canada. MedRxiv. https://doi.org/10.1101/2021. 07.05.21260050

50. Ong SWX, Chiew CJ, Ang LW et al Clinical and virological features of SARS-coV-2 variants of concern: a retrospective cohort study comparing B.1.1.7 (Alpha), B.1.315 (beta), and B.1.617.2 (delta). Preprint 07.06.1921. https://europepmc org/article/ppr/ppr356279 https://doi.org/10. 2139/ssrn.3861566.Zugegriffen: 10. Juli 2021

51. Sheikh A, McManin J, Taylor B, Robertson C, Public Health Scotlandand the EAVE II Collaborators (2021) SARS-CoV-2 Delta VOC in Scotland: demographics, risk of hospital admission, and vaccine effectiveness. Lancet 397:P2461-2462. https://doi.org/10.1016/S0140-6736(21)01358-1

52. Hoffmann M, Hoffmann-Winkler $H$, Krüger $N$ et al (2021) SARS-CoV-2 variant B.1.617 is resistant to Bamlanivimab and evades antibodies induced by infection and vaccination. Cell Rep 36:109415. https://doi.org/10.1016/j.celrep.2021.109415

53. Lopez Bernal J, Andrews N, Gower C et al (2021) Effectiveness of COVID-19 vaccines against the B.1.617.2 (Delta) variant. N Engl J Med. https://doi. org/10.1056/NEJMoa2108891

54. Edara VV, Lai L, Sahoo MKet al (2021) Infection and vaccine-induced neutralizing antibody responses to the SARS-CoV-2 B.1.617.1 variant. BioRxiv. https://doi.org/10.1101/2021.05.09.443299

55. Chia PY, Ong SWX, Chiew CJ et al (2021) Virological and serological kinetics of SARS-CoV-2 Delta variant vaccine- 1 breakthrough infections: a multi-center cohort study. MedRxiv. https:// www.medrxiv.org/content/10.1101/2021.07.28 21261295v1.full.pdf https://doi.org/10.1101/ 2021.07.28.21261295.Zugegriffen: 1. Aug. 2021

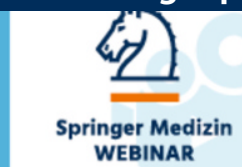

on Demand

LIVE-WEBINAR

ERC Reanimations-

leitlinien 2021

Prof. Dr. med. Bernd W. Böttiger

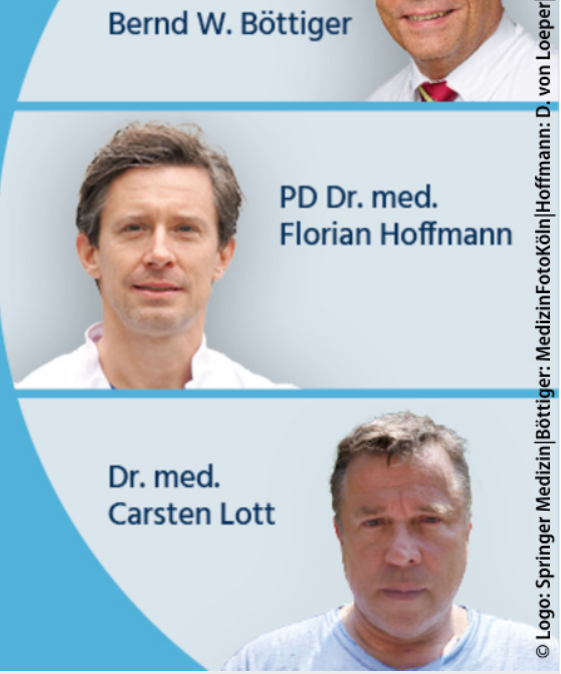

\section{Fragen zur Reanimation von Kindern?}

Ein Kind zu reanimieren ist auch für langjährige Ärtz*innen und Kolleg*innen des Rettungsdienstfachpersonals eine Herausforderungen. Oft stellen sich konkrete Fragen, die einen verunsichern. Lesen Sie hier kurz und knapp die Antworten von PD Dr. Florian Hoffmann auf einige Fragen Ihrer Kolleg*innen und die dazugehörigen Empfehlungen aus der ERC-Reanimationslinein 2021.

Das komplette Webinar zu den ERCReanimationsleitlinen

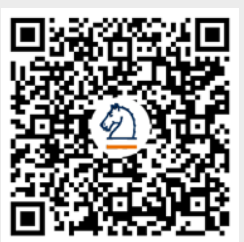

Im Webinar finden Sie die essentiellen Grundlagen der ERC-Reanimationsleitlinien zusammengefasst. Der Fokus liegt dabei auf Koronarthrombosen und Herzinfarkt und der der Renimation bei Kindern und Jugendlichen.

\section{Die Referenten:}

- Prof. Dr. B. W. Böttiger, Köln

- PD Dr. Florian Hoffman, München

- Dr. med Carsten Lott, Mainz

[URL: www.springermedizin.de/webinar-ercleitlinien]
Antworten aus 3 Fragen zum Webinar:

Erkennt der AED anhand des Körperwiderstands eine mögliche Reduktion der Energiezahl oder werden bei einem Säugling auch $360 \mathrm{~J}$ abgegeben?

>> Bisher erkennt der AED nur, ob K-Elektroden oder E-Elektroden angeschlossen sind. In Zukunft wird aber eine Berechnung nach Körperwiderstand realisiert werden können.

Gibt es eine Empfehlungen zum Zeitpunkt der Intubation unter CPR?

> Die Intubation ohne Effekt auf Outcome ist sehr in den Hintergrund gerückt und nur dann durchführbar, wenn alle notwendigen Maßnahmen laufen und ein Experte für Kinderintubation anwesend ist (>100-150 Intubationen).

Gibt es eine Evidenz zur Verwendung von Erwachsenen-Masken bei Kindern, im Sinne einer Full-Face-Alternative, falls kein Kinderbeutel verfügbar ist? >> Es gibt keine Evidenz aber Erfahrungen mit Drehen der Maske um $180 \mathrm{Grad}$, mit Einmalmasken mit aufblasbarem Rand kann auch mit Erwachsenen-Masken zumeist eine gute Ventilation erreicht werden. 